\title{
Diffeomorphism-invariant quantum field theories of connections in terms of webs
}

\author{
J Lewandowski†‡ and T Thiemann $\ddagger$ \\ $\dagger$ Instytut Fizyki Teoretycznej, Uniwersytet Warszawski, ul. Hoza 69, 00-681 Warsaw, Poland \\ ¥ Albert-Einstein-Institut, MPI für Gravitationsphysik, Schlaatzweg 1, 14473 Potsdam, Germany \\ E-mail: lewand@aei-potsdam.mpg.de \\ and thiemann@aei-potsdam.mpg.de
}

Received 11 January 1999

\begin{abstract}
In the canonical quantization of gravity in terms of the Ashtekar variables one uses paths in the 3-space to construct the quantum states. Usually, one restricts oneself to families of paths admitting only a finite number of isolated intersections. This assumption implies a limitation on the diffeomorphisms invariance of the introduced structures. In this work, using the previous results of Baez and Sawin, we extend the existing results to a theory admitting all the possible piecewise-smooth finite paths and loops. In particular, we (a) characterize the spectrum of the Ashtekar-Isham configuration space, (b) introduce spin-web states, a generalization of the spinnetwork states, (c) extend the diffeomorphism averaging to the spin-web states and derive a large class of diffeomorphism-invariant states and finally (d) extend the 3-geometry operators and the Hamiltonian operator.
\end{abstract}

PACS number: 0460D

\section{Introduction}

In the context of the canonical quantization of diffeomorphism-invariant theories of connections for a compact gauge group, in particular, quantum gravity in four spacetime dimensions, the quantum configuration space is coordinatized by certain elementary operators which could be called generalized Wilson functions. These functions are labelled in general by a set of paths in the manifold in question. It turns out that the precise nature of these paths has a deep impact on the structure on the resulting quantum theory. All the considerations in quantum gravity to date concern the case where the paths defining a given Wilson function can intersect only finitely many times. To ensure this, piecewise analyticity was often assumed. Therefore, in the following we loosely denote that case by 'the analytic category'. In 'the smooth category', however, the known results are sparse.

In this paper we extend the pioneering work by Baez and Sawin on this issue. Specifically, this paper is organized as follows. In section 2 we state the problem with the extension of results from the analytic to the smooth category and recall the results due to Baez and Sawin. Then we prove a master theorem which extends the results due to these authors and which is the key to sections 3-5. In section 3 we apply the master theorem to show how the results concerning the Ashtekar-Isham algebra can be immediately extended. In section 4 and 5 we define the notion of a spin web in analogy to the spin-nets of the analytic category in order to apply the master theorem to the extension of results by Ashtekar, Marolf, Mourão and the authors 
concerning diffeomorphism-invariant states of connections and associated diffeomorphisminvariant operators. Finally, in section 6 we apply the machinery of the previous sections to the operators that have proved to be elementary building blocks for the quantization of the gravitational field.

\section{The algebra of cylindrical functions on the space of connections}

This section is divided into three parts. In the first part we recall the basic notions of cylindrical functions on the space of distributional connections and inductive limit structures, and review the results that one obtains if one labels cylindrical functions by piecewise analytic, finitely generated graphs. In the second part we review the construction, due to Baez and Sawin, of a new label set, called 'webs', which are appropriate for the case where the loops defining the Wilson functions are only piecewise smooth and may intersect each other an infinite number of times. Finally, in the third part we prove the master theorem on which the rest of this paper is based.

\subsection{Preliminaries}

In the loop quantization of gravity as initiated by Ashtekar [1] and Jacobson, Rovelli and Smolin [2] and Gambini et al (see, e.g., [3] and references therein) through embedding general relativity into a Yang-Mills theory phase space and making use of Wilson loop variables as well as in a general framework for quantization of a diffeomorphism-invariant theory of connections with local degrees of freedom [4] one uses the algebra of cylindrical functions defined by the parallel transport maps as the main device [4-30]. The algebra, let us call it $\mathrm{Cyl}$, is a sub-algebra of the algebra of functions defined on a space of $G$ connections $\mathcal{A}$. The connections are defined on a bundle over a manifold $\Sigma$. For simplicity we assume here that the bundle is trivial $\dagger$ and we fix a global gauge (section). We are assuming that $G$ is compact and semi-simple. A complex-valued function $\Phi: \mathcal{A} \rightarrow \mathbb{C}$ is called cylindrical, if there are piecewise-smooth paths $p_{1}, \ldots, p_{n} \ddagger$ in $\Sigma$ and a function $\phi \in C^{0}\left(G^{n}\right)$ such that

$$
\Phi(A)=\phi\left(U_{p_{1}}(A), \ldots, U_{p_{n}}(A)\right)
$$

where $U_{p}(A) \in G$ represents the parallel transport along the path $p$ with respect to the connection $A$. To control the algebra it is useful to decompose Cyl into a union

$$
\mathrm{Cyl}=\cup_{w \in \mathcal{W}} \mathrm{Cyl}_{w}
$$

of sub-algebras that are easier to handle ( $\mathcal{W}$ is a labelling set and will be specified below). This decomposition is particularly convenient if it defines a so-called 'inductive limit', that is, if for every $w_{1}, w_{2} \in \mathcal{W}$, there exists $w^{\prime} \in \mathcal{W}$ such that

$$
\mathrm{Cyl}_{w_{1}}, \mathrm{Cyl}_{w_{2}} \subset \mathrm{Cyl}_{w^{\prime}} .
$$

One might take for $\mathcal{W}$ the set of all the finite families of piecewise-smooth paths in $\Sigma$ and associate to each finite family of paths $w$ the sub-algebra $\mathrm{Cyl}_{w} \subset \mathrm{Cyl}$ given by all the paths obtained from the elements of $w$ and their inverses by using the path product. That does define an inductive limit decomposition, however, given a general family of paths $w$, we do not know

\footnotetext{
$\dagger$ Most of the results concerning the gauge-invariant cylindrical functions can be easily generalized to a non-trivial bundle case $[6,7,9]$.

$\mp$ By a piecewise-smooth path we mean here a piecewise-smooth map $p:\left[t_{0}, t_{1}\right] \rightarrow \Sigma$ continues on the whole interval of $\left[t_{0}, t_{1}\right]$ such that its (one-sided) derivative never vanishes except for the constant path; here we are actually interested in equivalence classes of paths where two paths are equivalent iff they differ by an orientation-preserving piecewise-smooth diffeomorphism $\left[t_{0}, t_{1}\right] \mapsto\left[s_{0}, s_{1}\right]$.
} 
much about the corresponding $\mathrm{Cyl}_{w}$. An example of a sub-algebra $\mathrm{Cyl}_{w}$ we do know much about is the sub-algebra corresponding to a family of paths $w$ which is an embedded graph $†$. Then, $\mathrm{Cyl}_{w}$ can be identified with the algebra of functions $C^{0}\left(G^{n}\right)$ where $n$ equals the number of edges in $w$, via the map

$$
\mathcal{A} \ni A \quad \mapsto \quad\left(U_{e_{1}}(A), \ldots, U_{e_{n}}(A)\right) \in \mathcal{A}_{w} \subset G^{n} .
$$

where $e_{i}$ are the edges of $w$ and $\mathcal{A}_{w}$ is the image of that map. Such a map is defined for any family of paths, but in the case of a graph the map (2.4) is onto, therefore

$$
\mathcal{A}_{w}=G^{n}
$$

If one fixes an analytic structure on $\Sigma$ and defines $\mathrm{Cyl}_{(\omega)} \subset \mathrm{Cyl}$ to be the sub-algebra given by the piecewise-analytic paths, then the analytic graphs are enough to decompose $\mathrm{Cyl}_{(\omega)}$ into the following inductive limit sum:

$$
\cup_{\gamma} \mathrm{Cyl}_{\gamma}=\mathrm{Cyl}_{(\omega)},
$$

where the sum ranges over the set of piecewise-analytic graphs in $\Sigma$. That is true due to the following fact. Given two analytic graphs $\gamma, \gamma^{\prime}$ there is an analytic graph $\gamma^{\prime \prime}$ such that $\gamma^{\prime \prime} \geqslant \gamma, \gamma^{\prime}$, where the inequality relation is defined as follows. Two families $w, w^{\prime}$ of paths, are in the relation

$$
w \leqslant w^{\prime}
$$

if every path element of $w$ can be obtained from the elements of $w^{\prime}$ and their inverses by using the path product $\$$. Therefore, because of the composition rule of the parallel transports

$$
U_{p \circ p^{\prime}}=U_{p} U_{p^{\prime}}
$$

we have

$$
\mathrm{Cyl}_{\gamma_{1}}, \mathrm{Cyl}_{\gamma_{2}} \subset \mathrm{Cyl}_{\gamma^{\prime}} \cdot
$$

The above decomposition was key to the following issues:

- The development of integral calculus on $\mathrm{Cyl}_{(\omega)}[6,9]$.

- The characterization of the Gel'fand spectrum of the corresponding $C^{*}$ algebra when $G$ is compact $[5,6,8,10]$.

- Measure theory on the spectrum $[6,8,10]$.

- The introduction of differential calculus on the spectrum useful for introducing and studying the operators of quantum gravity [7].

- The introduction of spin-networks as the orthonormal basis with respect to the natural integral in $\mathrm{Cyl}_{(\omega)}[15,16]$.

Our goal now is to see to what extent the above applications can be generalized to the case of smooth paths.

\footnotetext{
$\dagger$ A graph is a finite family of one-dimensional, oriented sub-manifolds of $\Sigma$ such that every two can share only one or two points of the boundary. Given a graph, its elements are called edges. By an edge, we shall also mean an oriented 1-sub-manifold with boundary.

\$ Given two (unparametrized) paths $p_{1}$ and $p_{2}$ such that the end point of $p_{1}$ is a starting point of $p_{2}$, the path product $p_{2} \circ p_{1}$ is a path obtained by connecting the end of $p_{1}$ with the beginning point of $p_{2}$.
} 


\subsection{The Baez-Sawin framework}

The virtue of piecewise-analytic graphs is that their edges cannot intersect or overlap in an infinite number of distinct, isolated points or segments. This is the key to the definition of the algebra $\mathrm{Cyl}_{(\omega)}$ as an inductive limit since the union of two piecewise-analytic graphs (after a finite number of subdivisions) again defines a piecewise-analytic graph. This is no longer true when two graphs are just piecewise smooth, an example being given by the graph that consists of the union of two smooth curves which intersect in a Cantor set [31] or the intersection of the graph of the function $[0,1] \ni x \rightarrow \mathrm{e}^{-1 / x^{2}} \sin (1 / x)$ with the $x$-axis. The graphs are not both contained in the same graph. Thus, for the full algebra Cyl the graphs are not enough to provide an appropriate decomposition.

Recently, Baez and Sawin [17] introduced a suitable generalization of an embedded graph which they called a web.

For the purpose of this paper, it is enough to know the following properties of the webs due to Baez and Sawin:

(a) a web is a finite family of piecewise-smooth paths $e$ which do not self-intersect;

(b) for every web $w=\left\{e_{1}, \ldots, e_{n}\right\}$ the image of the map (2.4) is a Lie subgroup of $G^{n}$;

(c) for every finite family $w$ of smooth paths there exists a web $w^{\prime}$ such that $w \leqslant w^{\prime}$.

Thus, in contrast to the piecewise-analytic graphs which are labelled by their edges (i.e. paths which intersect at most at their endpoints) a Baez-Sawin web is labelled by the paths $e_{1}, \ldots, e_{n}$ which may overlap or intersect each other possibly an infinite number of times. Abusing the notation, we shall nevertheless denote the labels of a web by edges again to distinguish them from an arbitrary path. Henceforth, we denote by $\mathcal{W}$ the set of all the webs in $\Sigma$. We should also note that Baez-Sawin's $\mathcal{W}$ is preserved by the diffeomorphisms of $\Sigma$. The highly non-trivial result proved in [17] is that $\mathcal{W}$ indeed exists.

Due to property (c),

$$
\mathrm{Cyl}=\cup_{w \in \mathcal{W}} \mathrm{Cyl}_{w},
$$

and $\left(\mathrm{Cyl}_{w}\right)_{w \in \mathcal{W}}$ is a projective family. Point (b) gives us certain control on $\mathrm{Cyl}_{w}$. Our first result will tell us more about $\mathrm{Cyl}_{w}$.

We end this section with the Baez-Sawin definition of a web (for more details see [17]). A web is the (set) union of a finite number of families of edges, so-called tassels. First, to give the reader an intuitive picture, we explain how a graph gives rise to a web. Given a graph $\gamma$ subdivide each of its edges $e$ into two edges oriented in such a way that they end in the subdivision point. Denote the resulting graph by $w$. A vertex of $w$ is either a vertex of $\gamma$ or one of the subdivision points. Let $v$ be a vertex of $w$ which is also a vertex of $\gamma$. The set of edges of $w$ leaving $v$ forms a tassel based at $v$ and the subdivided graph $w$ is a web (a special case of a web).

In general, edges of a tassel are allowed to intersect at points different from their end points and can overlap. Given a family $T$ of edges, a point $q \in R(T) \dagger$ is a regular point of $T$ if there is a neighbourhood $\mathcal{U}$ of $q$ such that the intersection $R(T) \cap \mathcal{U}$ is an embedding of an open interval. A segment of that embedded interval we will call a regular segment. (In other words, if a curve $e \in T$ intersects $q$ then it intersects $q$ exactly once, and any other $e^{\prime} \in T$ either overlaps $e$ at $q$ or does not intersect $q$ at all.) Given a regular point $q$ of $T$ its type is the set of the elements of $T$ which overlap at $q$.

$\dagger$ The range $R(T)$ of a family of curves $T$ is the union of the ranges of each element of $T$. 
General definition of a web. A a family of edges is a tassel if it has the following properties.

(a) All the edges of $T$ begin at the same point; we call it the base point of $T$.

(b) The edges $e_{I}, I=1, \ldots, k$, of $T$ can be parametrized in such a way that

$$
e_{I}(t)=e_{J}(s) \quad \Rightarrow \quad t=s .
$$

(c) Two edges of $T$ that intersect at a point different from the base point $p$ intersect in every neighbourhood of $p$.

(d) Any type which occurs at some point $q \in R(T)$ occurs in every neighbourhood of the base point $p$.

\subsection{Holonomic independence of the curves in a Baez-Sawin web}

Paths $p_{1}, \ldots, p_{n}$ are holonomically independent if for every $\left(g_{1}, \ldots, g_{n}\right) \in G^{n}$ there is a smooth connection $A \in \mathcal{A}$ such that

$$
\left(U_{p_{1}}(A), \ldots, U_{p_{n}}(A)\right)=\left(g_{1}, \ldots, g_{n}\right) \in G^{n} .
$$

As we already indicated in section 2, the edges of any graph $\gamma$ are holonomically independent, hence the corresponding $\mathrm{Cyl}_{\gamma}$ can be identified with the whole of $C^{0}\left(G^{n}\right)$. However, the results of [17] only show that for an $n$-element web $w$ the set $\mathrm{Cyl}_{w}$ can be identified with $C^{0}\left(G_{w}\right)$ where a priori $G_{w}$ can be any Lie subgroup of $G^{n}$ that may even vary with $w$.

Our master result is that it continues to hold that $G_{w}=G^{n}$ for the case that $G$ is semisimple.

Theorem 2.1 (Master theorem). Let $\left\{p_{1}, \ldots, p_{n}\right\}$ be a finite family of paths which satisfies the properties $(a)$ and $(b)$ above. Then the paths are holonomically independent.

Proof. For $n=1$ the assertion is obvious. For $n=2$ we need to note that since $e_{1} \neq e_{2}$, there is a point $x \in R\left(e_{1}\right)$ such that a neighbourhood $\mathcal{U}_{1}$ of $x$ does not intersect $e_{2}$ and a similar point $y \in R\left(e_{2}\right)$ and a neighbourhood $\mathcal{U}_{2}$. The neighbourhoods may be chosen not to intersect each other. Therefore, given any $\left(g_{1}, g_{2}\right) \in G^{2}$ one can easily construct a single connection $A$ such that $U_{e_{1}}(A)=g_{1}$ and $U_{e_{2}}(A)=g_{2}$.

Suppose now that the theorem is true for every $n \leqslant k$ and consider the case when $n=k+1 \geqslant 3$. Denote by $\boldsymbol{a}_{w}$ the Lie sub-algebra of the Lie algebra of $G^{n}$ corresponding to the subgroup $\mathcal{A}_{w}=G_{w} \subset G^{n}$ obtained as the image of the map

$$
\mathcal{A} \ni \mapsto\left(U_{e_{1}}(A), \ldots, U_{e_{n}}(A)\right) \in G^{n} .
$$

It suffices to prove that $\boldsymbol{a}_{w}$ coincides with the whole Lie algebra of $G^{n}$. Since, according to our assumption, the theorem holds for $w^{\prime}=w \backslash\left\{e_{i}\right\}$ for any edge $e_{i}$, it is enough to show that for every $a \in \boldsymbol{g}$ in the Lie algebra of $G$ the $n$-tuple $(a, 0, \ldots, 0)$ is in $\boldsymbol{a}_{w}$ since $\boldsymbol{a}_{w}$ is a vector space (owing to Baez and Sawin).

Since $G$ is semi-simple, for any $a \in \boldsymbol{g}$ there are $b, b^{\prime} \in \boldsymbol{g}$ such that $\left[b, b^{\prime}\right]=a$. By an induction assumption, we can freely specify $n$ of the entries $b_{i}$ of an element $\left(b_{1}, \ldots, b_{n+1}\right)$ of the Lie algebra $\boldsymbol{a}_{w}$, however, the last entry may depend on the other $n$ entries. Let us choose freely $b_{1}=b, b_{3}=\cdots=b_{n+1}=0$ and $b_{1}^{\prime}=b^{\prime}, b_{2}^{\prime}=b_{4}=\cdots=b_{n+1}^{\prime}=0$. Then there exist certain $\tilde{b}, \tilde{b}^{\prime} \in \boldsymbol{g}$ which may depend on the already specified $b_{i}$ and $b_{i}^{\prime}$, respectively, such that

$$
(b, \tilde{b}, 0, \ldots, 0),\left(b^{\prime}, 0, \tilde{b}^{\prime}, 0, \ldots, 0\right) \in \boldsymbol{a}_{w} .
$$

Because $\boldsymbol{a}_{w}$ is closed with respect to the commutator (again owing to Baez and Sawin), we have

$$
\left[(b, \tilde{b}, 0, \ldots, 0),\left(b^{\prime}, 0, \tilde{b}^{\prime}, 0, \ldots, 0\right)\right]=(a, 0, \ldots, 0) \in \boldsymbol{a}_{w},
$$

which completes the proof. 


\section{Immediate implications of the master theorem}

In this section we list the immediate consequences of theorem 2.1 concerning the issue of (Mandelstam) group identities which are an important building block in the construction of the Ashtekar-Isham algebra [5], the spectrum of that algebra and a natural uniform measure thereon.

\subsection{Group identities}

The issue is the following.

Suppose that $k$ complex-valued functions $\phi_{1}, \ldots, \phi_{k}$ defined on $G^{n}$ satisfy a group identity

$$
P\left(\phi_{1}, \ldots, \phi_{k}\right)=0 \quad \forall\left(g_{1}, \ldots, g_{n}\right) \in G^{n},
$$

where $P$ is a complex-valued function of $k$ complex variables which is characteristic for the group $G$. Examples of such identities are the famous Mandelstam identities which for the group $G=S U(2)$ are given by

$$
\operatorname{tr}(g) \operatorname{tr}\left(g^{\prime}\right)=\operatorname{tr}\left(g g^{\prime}\right)+\operatorname{tr}\left(g\left(g^{\prime}\right)^{-1}\right) .
$$

Now take any $n$-tuple of paths $p_{1}, \ldots, p_{n}$ in $\Sigma$ and consider the following cylindrical functions defined on $\overline{\mathcal{A}}$ :

$$
\Phi_{i}(A)=\phi_{i}\left(U_{p_{1}}(A), \ldots, U_{p_{n}}(A)\right), \quad A \in \overline{\mathcal{A}}, \quad i=1, \ldots, k .
$$

Obviously these cylindrical functions then satisfy the identity

$$
P\left(\Phi_{1}, \ldots, \Phi_{k}\right)=0 \quad \forall A \in \mathcal{A} .
$$

Thus, we see that every group identity gives rise to one identity on the algebra Cyl.

The question that arises is whether every identity that holds on the algebra Cyl (or one of its sub-algebras) results from a group identity.

Owing to the master theorem, the answer turns out to be affirmative (one could not prove this result without knowing theorem 2.1).

Indeed, consider any system of cylindrical functions $\Phi_{1}, \ldots, \Phi_{k} \in$ Cyl satisfying some identity

$$
P\left(\Phi_{1}, \ldots, \Phi_{k}\right)=0
$$

Any of the cylindrical functions is defined by (2.1) with respect to some finite set of paths in $\Sigma$. There exists a web $w=\left\{p_{1}, \ldots, p_{n}\right\}$ whose range contains all the paths used to define the functions $\Phi_{1}, \ldots, \Phi_{k}$. Thus $\Phi_{i}=p_{w}^{*} \phi_{i}$ for some functions $\phi_{i}$ defined on the image $\mathcal{A}_{w}=G_{w}=p_{w}(A)$ of (2.13). Consequently, the functions $\phi_{i}$ satisfy the corresponding identity (3.1) on all of $G_{w}$. However, according to theorem 2.1 we have $G_{w}=G^{n}$ where $n$ is the number of the edges of $w$. Thus the identity on cylindrical functions came from a group identity. Notice that, in particular, the path identities on cylindrical functions

$$
U_{p}(A) U_{q}(A)=U_{p \circ q}(A), \quad U_{p \circ p^{-1}}=1_{G}
$$

for any two paths $p, q$ and the Mandelstam identities, which for $S U$ (2) take the form

$$
\operatorname{Tr} U_{\alpha \circ \beta}+\operatorname{Tr} U_{\alpha \circ \beta^{-1}}=\operatorname{Tr} U_{\alpha} \operatorname{Tr} U_{\beta},
$$

decline from a group identity. 
A related question is 'when are two paths $p$ and $q$ holonomically equivalent?' That is, for which paths $p$ and $q$ does the identity

$$
U_{p}(A)=U_{q}(A)
$$

hold on $\mathcal{A}$ (i.e. for all $A \in \mathcal{A}$ )?

Let paths $p$ and $q$ be holonomically equivalent. Choose a web $w=\left\{p_{1}, \ldots, p_{n}\right\}$ such that $\{p, q\} \leqslant w$. Decompose $p, q$ into the path products of the elements of $w$ and their inverses:

$$
\begin{aligned}
& p=e_{i(1)}^{\kappa(1)} \circ \cdots \circ e_{i(N)}^{\kappa(N)} \\
& q=e_{j(1)}^{l(1)} \circ \cdots \circ e_{j(M)}^{l(M)} .
\end{aligned}
$$

Suppose that, in this decomposition, all the sequences of the form $e_{I} \circ e_{I}^{-1}$ have been cancelled.

The properties of the parallel transport, the holonomic equivalence of the paths gives an identity

$$
g_{i(1)}^{\kappa(1)} \cdots g_{i(N)}^{\kappa(N)}=g_{j(1)}^{l(1)} \cdots g_{j(M)}^{l(M)}
$$

true for every $\left(g_{1}, \ldots, g_{n}\right) \in G_{w}$.

However, precisely because theorem 2.1 holds, $G_{w}=G^{n}$ which enables us, at least when $G$ is non-Abelian and compact, to conclude that modulo the cancellation of pairs $g_{i} g_{i}^{-1}$,

$$
N=M, \quad i(r)=j(r), \quad \kappa(r)=l(r) .
$$

That is for a non-Abelian compact Lie group, paths $p$ and $q$ are holonomically equivalent iff $p=q$ modulo cancellation of the segments of the form $\cdots \circ e \circ e^{-1} \circ \cdots$. (Here we are relying on a related fact from the theory of compact non-Abelian groups [18].) Notice that we could not conclude this if $G_{w}$ was a proper sub-Lie group of $G^{n}$.

\subsection{Spectrum of the Ashtekar-Isham algebra}

The main motivation for raising the question about the precise image of the map (2.4) was the wish to give a characterization of the Ashtekar-Isham quantum configuration space which in the analytical category turned out to be rather straightforward. Recall, that when $G$ is compact, we define in the algebra of cylindrical functions Cyl a norm

$$
\|\Phi\|:=\sup _{A \in \mathcal{A}}|\Phi(A)| \text {. }
$$

The completion of Cyl with respect to that norm is a $C^{*}$ algebra. Its Gel'fand spectrum $\overline{\mathcal{A}}$ has been promoted by Ashtekar and Isham to the role of the quantum configuration space for a connection theory. According to the results of $[6-8,10]$ an element $\bar{A}$ of the spectrum of Cyl can be identified naturally with a family of points $\left(\bar{A}_{w}\right)_{w \in \mathcal{W}}$ which satisfies a certain consistency condition. Namely, given two webs $w \leqslant w^{\prime}$, the projections $p_{w}: \mathcal{A} \rightarrow \mathcal{A}_{w}$ and $p_{w^{\prime}}: \mathcal{A} \rightarrow \mathcal{A}_{w^{\prime}}$ defined in (2.4) determine uniquely a projection

$$
p_{w w^{\prime}}: \mathcal{A}_{w^{\prime}} \rightarrow \mathcal{A}_{w}, \quad \text { such that } \quad p_{w w^{\prime}} \circ p_{w^{\prime}}=p_{w} .
$$

The consistency condition satisfied by $\left(\bar{A}_{w}\right)_{w \in \mathcal{W}}$ is that for every pair of webs $w \leqslant w^{\prime}$,

$$
p_{w w^{\prime}} \bar{A}_{w^{\prime}}=\bar{A}_{w} \text {. }
$$

More precisely, there is a one-to-one correspondence between the set of solutions of the consistency condition and the spectrum. To solve the consistency conditions one considers a map $U(\bar{A})$ which assigns to every path $p$ an element of $G$,

$$
p \mapsto U(\bar{A})_{p} \in G,
$$


such that

$$
U(\bar{A})_{p \circ q}=U(\bar{A})_{p} U(\bar{A})_{q}, \quad \text { and } \quad U(\bar{A})_{p^{-1}}=\left(U(\bar{A})_{p}\right)^{-1} .
$$

Indeed, a map $U(\bar{A})$ defines a family $\left(\bar{A}_{w}\right)_{w \in \mathcal{W}}$ by

$$
\bar{A}_{w}:=\left(U(\bar{A})_{p_{1}}, \ldots, U(\bar{A})_{p_{n}}\right) \in G^{n}=\mathcal{A}_{w}
$$

where $w=\left\{p_{1}, \ldots, p_{n}\right\} \dagger$. It is not hard to see that the result solves the consistency conditions and that every solution can be obtained in that way.

The algebra $\mathrm{Cyl}$ contains a sub-algebra $\operatorname{Cyl}(\mathcal{A} / \mathcal{G})$ of gauge-invariant cylindrical functions. Also the results of $[6-8,10]$ apply, with the graphs replaced by webs. In particular, the spectrum $\overline{\mathcal{A} / \mathcal{G}}$ is

$$
\overline{\mathcal{A} / \mathcal{G}}=\overline{\mathcal{A}} / \overline{\mathcal{G}}
$$

where $\overline{\mathcal{G}}$ is the group of all the maps from $\Sigma$ into $G$, the Gel'fand completion of the gauge group acting in $\overline{\mathcal{A}}$.

\subsection{A natural measure}

A measure $\mu_{0}$ on the spectrum $\overline{\mathcal{A}}$ is defined by any family of measures $\left(\mathcal{A}_{w}, \mu_{w}\right)_{w \in \mathcal{W}}$ such that given a cylindrical function $\Phi$ in the form (2.1), the integral

$$
\int \phi \mathrm{d} \mu_{w}=: \int_{\overline{\mathcal{A}}} \mathrm{d} \mu_{0} \Phi
$$

is independent of the choice of the web $w$. By the same arguments as those used in [6], if $G$ is compact and we choose for each $\mu_{w}$ the probability Haar measure, the resulting family of measures does satisfy the consistency condition and defines a natural, diffeomorphisminvariant measure on $\overline{\mathcal{A}}$. The resulting integral restricted to the sub-algebra of gauge-invariant elements of Cyl coincides with the natural measure defined in $[7,8]$. This result was derived previously by Baez and Sawin and our theorem 2.1 mainly simplifies the argument.

\section{Spin-webs and diffeomorphism averaging}

With the natural measure, the space of the cylindrical functions is naturally completed to become the Hilbert space

$$
\mathcal{H}:=L^{2}\left(\overline{\mathcal{A}}, \mathrm{d} \mu_{0}\right)
$$

The aim of this section is to find a generalization of the spin-network states [15,20] constructed from graphs. The spin-networks gave an orthogonal decomposition of the Hilbert completion of the subspace of $\mathcal{H}$ given by the piecewise-analytic paths and were used to define the averaging with respect to all the diffeomorphisms of $\Sigma$.

$\dagger$ It is here where we need theorem 2.1. When $U$ varies, the points $\bar{A}_{w}$ fill all of $G^{n}$. Due to $G^{n}=\mathcal{A}_{w}$, every $U$ defines a point in $\mathcal{A}_{w}$ 


\subsection{Spin-webs}

Given a web $w$ we associate a Hilbert subspace $\mathcal{H}_{w}$, the Hilbert completion of $\mathrm{Cyl}_{w}$. That subspace is isometric with $L^{2}\left(G^{n}\right)$. That identification provides an orthogonal decomposition given by $L^{2}(G)=\oplus_{j} V_{j}$ where $V_{j}$ are subspaces labelled by the irreducible representations of $G$. In this way, to each labelled web $(w, j)$, that is, a pair consisting of a web $w$ and a labelling $j$ of the edges of $w$ by irreducible representations, we associate a finite-dimensional subspace $V_{w, j} \subset \mathcal{H}_{w}$ called a spin-web space $\dagger$. The spin-web subspaces $H_{w j}$ of $\mathcal{H}_{w}$ corresponding to all possible labellings $j$ span $\mathcal{H}_{w}$, are finite dimensional and for different labellings they are orthogonal.

If $j(p)=0$ for an edge $p$ of a labelled web $(w, j)$, then we can consider another labelled spin-web $\left(w^{\prime}, j^{\prime}\right)$ given by removing the edge $p$ from $w$ and maintaining the labelling of the remaining edges. Obviously, the corresponding spin-web spaces are equal. In the case of graphs, that degeneracy can be removed by admitting only non-trivial representations for the labelling of its edges and vertices. However, in the case of the proper webs, that is not enough and still there are spin-web spaces associated to different webs $R\left(w^{\prime}\right) \neq R(w)$ such that $V_{w j}$ fails to be orthogonal to $V_{w^{\prime} j^{\prime}}$. Since that is an important difficulty in diffeomorphism averaging of the spin-webs, we illustrate it by the following example.

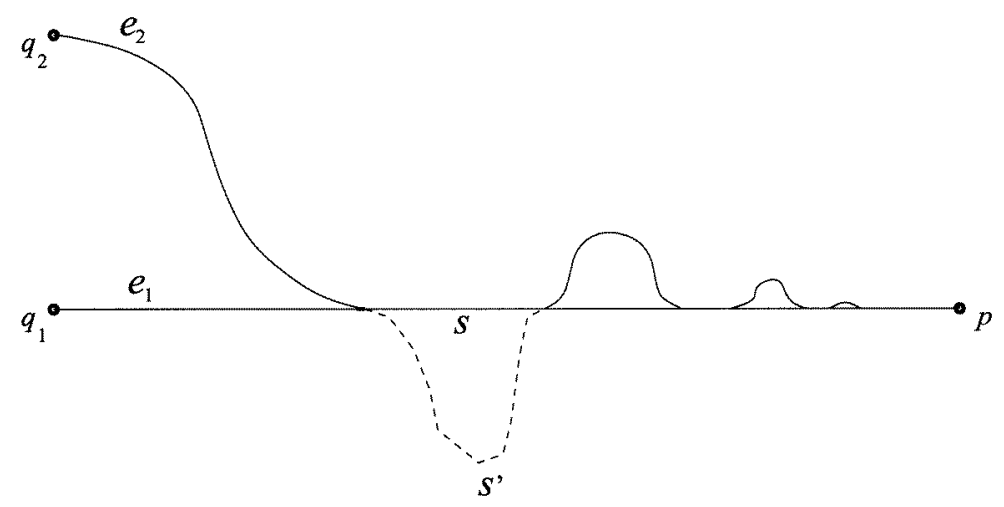

Figure 1. The edge $e_{1}$ is the horizontal line, whereas the edge $e_{2}$ consists of infinitely many bumps up the horizontal line connected with the horizontal segments (only four bumps of $e_{2}$ are visible in the figure. The edges $e_{1}^{\prime}$ and $e_{2}^{\prime}$ are obtained by replacing the full segment $s$ by the broken segment $s^{\prime}$

Example. The first web is $w=\left\{e_{1}, e_{2}\right\}$. This is just one tassel of a base point $p$. The second web consists of the deformed edges. It is $w=\left\{e_{1}^{\prime}, e_{2}^{\prime}\right\}$ (see figure 1). For the labellings of $w$ and $w^{\prime}$, respectively, we take $j\left(e_{1}\right)=j\left(e_{2}\right)=j^{\prime}\left(e_{1}^{\prime}\right)=j^{\prime}\left(e_{2}^{\prime}\right)=\frac{1}{2}$. Our claim is that the corresponding spin-web spaces $V_{w, j}$ and $V_{w^{\prime}, j^{\prime}}$ are not orthogonal. To see this, consider $a$ web $\tilde{w}$ obtained from $w$ and $w^{\prime}$ by subdividing their edges, such that the segments $s$ and $s^{\prime}$ become path products of entire edges of $\tilde{w}$ (see figure 2). The spin-web space $V_{w, j}$ is no longer a spin-web space with respect to the web $\tilde{w}$. It admits the non-vanishing projection on the spin-web space given by the labelling $\tilde{j}$ of $\tilde{w}$ which takes the value 0 on the segments of $s$ and $s^{\prime}$, and $\frac{1}{2}$ otherwise. The same is true for the spin-web $V_{w^{\prime}, j^{\prime}}$.

$\dagger$ With an edge $p$ of a web $w$ and an irreducible representation $j(p)$ one associates the linear span $V_{p, j(p)}$ of the functions $f(A)=\mathcal{D}^{(j(p))}{ }_{N}^{M}(U(A))$, where $\mathcal{D}^{(j)}{ }_{N}^{M}(U)$ denotes an $(M, N)$ entry of the matrix corresponding to an element $U \in G$ in the $j$ th representation and with respect to some fixed basis in the fundamental representation space. Then, $V_{w, j}=\oplus_{p} V_{p, j(p)}$, with $p$ running through the edges of $w$. 


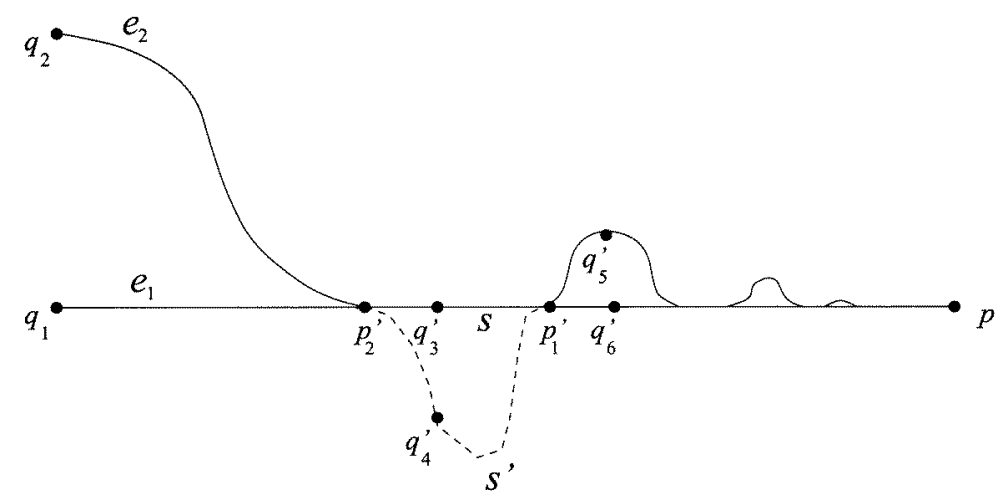

Figure 2. The web $\tilde{w}$ consists of three tassels. The first tassel is based at the point $p$ and consists of the segments connecting $p$ with the points $q_{5}^{\prime}$ and $q_{6}^{\prime}$. The second tassel is based at the point $p_{1}^{\prime}$. It consists of the segments connecting $p_{1}^{\prime}$ with the points $q_{3}^{\prime}, q_{4}^{\prime}, q_{5}^{\prime}, q_{6}^{\prime}$. The third tassel is the set of the segments connecting the point $p_{2}^{\prime}$ with the points $q_{1}, q_{2}, q_{3}^{\prime}, q_{4}^{\prime}$.

The lesson the above example teaches us is that a smooth deformation of a segment $s$ in $(w, j)$ such that it no longer overlaps the remaining part of $w$ does not necessarily result in a labelled spin-web $\left(w^{\prime}, j^{\prime}\right)$ such that $V_{w^{\prime}, j^{\prime}}$ and $V_{w, j}$ are orthogonal to each other. This would not be true if we were working in the analytical category and with spin-networks $[15,16]$. We could also see how two different webs define a refined, bigger one.

Our goal now is to show that there exist certain 'non-degenerate' spaces $V_{w, j}$ with the property that they are orthogonal to every other space $V_{w^{\prime}, j^{\prime}}$ whenever the ranges of the webs do not coincide, whether $\left(w^{\prime}, j^{\prime}\right)$ is also non-degenerate or not.

Definition 4.1. Consider a web $w$ and a labelling $j$ of its edges with irreducible and non-trivial representations. Consider a regular point $x$ (see section 2.2) of $w$ and let the type of $x$ be such that the edges $e_{I_{1}}, \ldots, e_{I_{k}} \in w$ are overlapping at $x$. We will say that $x$ is a non-degenerate point whenever the trivial representation does not emerge in the decomposition of the tensor product of the representations $j\left(e_{I_{1}}\right), \ldots, j\left(e_{I_{k}}\right)$. Given a labelled web $(w, j)$, if every regular point is non-degenerate, then we will say that $(w, j)$ is non-degenerate.

We then have the following result.

Theorem 4.1. Suppose $V_{w, j}$ is the spin-web space assigned to a non-degenerate labelled web $(w, j)$ and the spin-web space $V_{w^{\prime}, j^{\prime}}$ assigned to $\left(w^{\prime}, j^{\prime}\right)$ is not orthogonal to $V_{w, j}$; assume also that $j^{\prime}\left(e^{\prime}\right) \neq 0$ for every $e^{\prime} \in w^{\prime}$; then:

(a) The labelled spin-web $\left(w^{\prime}, j^{\prime}\right)$ is also non-degenerate;

(b) The ranges of the spin-webs coincide,

$$
R(w)=R\left(w^{\prime}\right)
$$

(c) There is a web $w^{\prime \prime} \geqslant w, w^{\prime}$ which can be obtained from each of the webs $w, w^{\prime}$ by subdividing its edges.

Proof of theorem 4.1. The key fact is the existence of a web $w^{\prime \prime}$ such that

$$
w^{\prime \prime} \geqslant w, w^{\prime}
$$

One has to remember (see the example of section 4.1), however, that for proper webs, that is for webs which are not just graphs, given the spin-web space $V_{w, j}$ and the bigger web $w^{\prime \prime}$, the 
space $V_{w, j}$ is not in general one of the spaces $V_{w^{\prime \prime}, j^{\prime \prime}}$ with some labelling $j^{\prime \prime}$. All that we know is that in general

$$
V_{w, j}, V_{w^{\prime}, j^{\prime}} \subset \oplus_{j^{\prime \prime}} V_{w^{\prime \prime}, j^{\prime \prime}},
$$

where $j^{\prime \prime}$ is some finite set of labellings. Since the spin-web spaces $V_{w, j}$ and $V_{w^{\prime}, j^{\prime}}$ are not orthogonal to each other, there is a labelling $j_{0}^{\prime \prime}$ of the web $w^{\prime \prime}$ such that both the projections

$$
V_{w, j} \rightarrow V_{w^{\prime \prime}, j_{0}^{\prime \prime}}, \quad \text { and } \quad V_{w^{\prime}, j^{\prime}} \rightarrow V_{w^{\prime \prime}, j_{0}^{\prime \prime}}
$$

are not trivial. An easy observation is the following

Lemma 4.1. Let $(w, j)$ and $\left(w^{\prime}, j^{\prime}\right)$ be as in the hypothesis above; there is a web $w_{0}^{\prime \prime} \geqslant w, w^{\prime}$ which can be obtained by sub-dividing the edges of $w^{\prime}$.

Proof of lemma 4.1. Let $w^{\prime \prime}$ be a web such that $w^{\prime \prime} \geqslant w, w^{\prime}$. Let $w_{1}, w_{2} \subset w^{\prime \prime}$ be webs which consist of the segments of the edges of $w$, and, respectively, $w^{\prime}$. Thus we can write,

$$
\begin{aligned}
w_{1} & =\left\{e_{1}^{\prime \prime}, \ldots, e_{k}^{\prime \prime}, a_{1}^{\prime \prime}, \ldots, a_{n}^{\prime \prime}\right\}, \\
w_{2} & =\left\{e_{1}^{\prime \prime}, \ldots, e_{k}^{\prime \prime}, b_{1}^{\prime \prime}, \ldots, b_{m}^{\prime \prime}\right\}, \\
w^{\prime \prime} & =\left\{e_{1}^{\prime \prime}, \ldots, e_{k}^{\prime \prime}, a_{1}^{\prime \prime}, \ldots, b_{m}^{\prime \prime}, c_{1}^{\prime \prime}, \ldots\right\},
\end{aligned}
$$

that is the webs $w_{1}$ and $w_{2}$ share the edges $e_{I}^{\prime \prime}$, the edges $a_{I}^{\prime \prime}$ denote those of the edges of $w_{1}$ which are not contained in $w_{2}$, whereas $b_{J}^{\prime \prime}$ denote the edges of $w_{2}$ not contained in $w_{1}$. The first observation is, that the web obtained from the third web above by removing the extra edges $c_{I}$, that is,

$$
w_{0}^{\prime \prime}:=\left\{e_{1}^{\prime \prime}, \ldots, e_{k}^{\prime \prime}, a_{1}^{\prime \prime}, \ldots, b_{m}^{\prime \prime}\right\}
$$

also satisfies $w_{0}^{\prime \prime} \geqslant w, w^{\prime}$. It is constructed from segments of the edges of $w$ and $w^{\prime}$. Consider now the labelling $j_{0}^{\prime \prime}$ of (4.5). Since the first projection is not trivial and due to the nondegeneracy of $(w, j)$ we have

$$
j_{0}^{\prime \prime}\left(e_{I}^{\prime \prime}\right), j_{0}^{\prime \prime}\left(a_{J}^{\prime \prime}\right) \neq 0, \quad \text { and } \quad j_{0}^{\prime \prime}\left(b_{I}^{\prime \prime}\right)=0 .
$$

On the other hand, because the second projection is also non-trivial, we have

$$
j_{0}^{\prime \prime}\left(a_{J}^{\prime \prime}\right)=0 .
$$

Therefore, the extra elements $a_{I}^{\prime \prime}$ of the web $w_{1}$ do not exist, and we finally have

$$
\begin{aligned}
& w_{1}=\left\{e_{1}^{\prime \prime}, \ldots, e_{k}^{\prime \prime}\right\}, \\
& w_{0}^{\prime \prime}=w_{2}=\left\{e_{1}^{\prime \prime}, \ldots, e_{k}^{\prime \prime}, b_{1}^{\prime \prime}, \ldots, b_{m}^{\prime \prime}\right\} .
\end{aligned}
$$

This completes lemma 4.1.

From now on, let

$$
w^{\prime \prime}=w_{0}^{\prime \prime} \geqslant w, w^{\prime}
$$

be the web given by lemma 4.1. Eventually, we will show that if spin-web spaces $V_{w, j}$ and $V_{w^{\prime}, j^{\prime}}$ are not orthogonal to each other, then the non-degeneracy of $(w, j)$ implies the nondegeneracy of $\left(w^{\prime}, j^{\prime}\right)$. Together with lemma 4.1 that will complete the proof. However, before that we need two intermediate lemmas.

Consider two webs $w \leqslant w^{\prime \prime}$. An edge $e \in w$ is the path product of the elements of $w^{\prime \prime}$,

$$
e=e_{k}^{\prime \prime} \circ \cdots \circ e_{1}^{\prime \prime},
$$

where $e_{1}^{\prime \prime} \in w^{\prime \prime}$ is the one that contains the base point of $e$. We will see below that given a labelling $j$ of the web $w$, in the decomposition of the corresponding spin-web space $V_{w, j}$ into the spaces $V_{w^{\prime \prime}, j^{\prime \prime}}$ the labelling $j^{\prime \prime}$ of the beginning segment $e_{1}^{\prime \prime}$ coincides with the labelling $j$ of the corresponding edge $e$, for every edge $e \in w$. 
Lemma 4.2. Let $(w, j)$ be a labelled $w e b$ and $w^{\prime \prime} \geqslant w$; consider an edge $e \in w$; let $e_{1}^{\prime \prime} \in w^{\prime \prime}$ be the segment of e containing its base point; then

$$
V_{w, j} \subset \oplus_{j^{\prime \prime}: j^{\prime \prime}\left(e_{1}^{\prime \prime}\right)=j(e)} V_{w^{\prime \prime}, j^{\prime \prime}} .
$$

Proof of lemma 4.2. The spin-web space $V_{w, j}$ is spanned by the cylindrical functions of the form

$$
\otimes_{e \in w} \mathcal{D}^{(j(e))^{m(e)}}\left(U_{e}\right)
$$

Each such function is expressed by the parallel transports along the elements of $w^{\prime \prime}$ through the decomposition

$$
\left.\mathcal{D}^{(j(e))_{n(e)}^{m(e)}}\left(U_{(} e\right)\right)=\left(\mathcal{D}^{\left(j\left(e_{k}^{\prime \prime}\right)\right)}\left(U_{e_{k}^{\prime \prime}}\right) \cdots \mathcal{D}^{\left(j\left(e_{1}^{\prime \prime}\right)\right)}\right)\left(U_{e_{1}^{\prime \prime}}\right)_{n(e)}^{m(e)}
$$

where $j\left(e_{1}^{\prime \prime}\right)=\cdots=j\left(e_{k^{\prime \prime}}^{\prime \prime}\right)=j(e)$, since $\mathcal{D}$ is a representation and $U_{e_{k}^{\prime \prime}} \cdots U_{e_{1}^{\prime \prime}}=U(e)$.

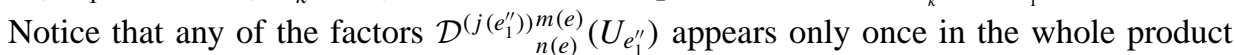
(4.15). To see this, we note that different edges $e_{1}$ and $e_{2}$ may share the same segment $e_{I}^{\prime \prime}$. However, by the definition of a web $w$ ([17], see also section 2.2), a segment of an edge $e_{1}$ of $w$ connected to the base point of a tassel in $w$ cannot be contained in a different edge $e_{2}$ of the same web.

Lemma 4.3. Suppose $(w, j)$ is a non-degenerate labelled web and $\left(w^{\prime}, j^{\prime}\right)$ is another labelled web such that the associated spin-web spaces $V_{w, j}$ and $V_{w^{\prime}, j^{\prime}}$ are not orthogonal; then $\left(w^{\prime}, j^{\prime}\right)$ is also non-degenerate.

Proof of lemma 4.3. Consider a regular point $x^{\prime}$ of the web $w^{\prime}$. Denote by $e_{1}^{\prime}, \ldots, e_{k}^{\prime}$ the edges of $w^{\prime}$ which overlap at $x^{\prime}$. What we have to show is that the tensor product of the corresponding representations $j^{\prime}\left(e_{1}^{\prime}\right), \ldots, j^{\prime}\left(e_{l}^{\prime}\right)$ does not contain the trivial representation. Use the web $w^{\prime \prime}$ of lemma 4.1 again and consider the labelling $j_{0}^{\prime \prime}$ of (4.5). Let the sub-webs $w_{1}, w_{2} \subset w^{\prime \prime}$ be defined as in (4.10). One of the edges of $w^{\prime \prime}$ is the segment of the edge $e_{1}^{\prime}$ which contains its base point of the corresponding tassel. Denote it by $e^{\prime \prime}$. Notice, that according to lemma 4.2

$$
j_{0}^{\prime \prime}\left(e^{\prime \prime}\right)=j^{\prime}\left(e_{1}^{\prime}\right) \neq 0
$$

Comparison with (4.8) shows that $e^{\prime \prime}$ is one of the edges $e_{I}^{\prime \prime}$, say $e_{1}^{\prime \prime}$, of the sub-web obtained by subdividing the edges of $w^{\prime}$. The same argument applies to each of the edges overlapping at $x^{\prime}$. Denote the corresponding segments by $e_{1}^{\prime \prime}, \ldots, e_{l}^{\prime \prime} \in w_{1} \subset w^{\prime \prime}$. Since they are segments of edges $e_{I}^{\prime}$ of a single tassel and each of them contains the base point, there is a regular point $x^{\prime \prime}$ of $w^{\prime \prime}$ such the edges $e_{1}^{\prime \prime}, \ldots, e_{l}^{\prime \prime}$ overlap at $x^{\prime \prime}$ and $x^{\prime \prime}$ is not intersected by any other of the edges of $w^{\prime \prime}$. However, the tensor product of the representation $j_{0}^{\prime \prime}\left(e_{1}^{\prime \prime}\right), \ldots, j_{0}^{\prime \prime}\left(e_{l}^{\prime \prime}\right)$ does not give the trivial one, because the labels $j^{\prime \prime}\left(e_{I}\right)$ come from the decomposition of the nondegenerate labelled web $(w, j)$ into the spin web spaces $V_{w^{\prime \prime}, j^{\prime \prime}}$ and the projection onto $V_{w^{\prime \prime}, j_{0}^{\prime \prime}}$ is non-trivial. This concludes the proof of lemma 4.3 and the proof of theorem 4.1.

What we have learnt from this section is that despite the difficulties shown in the example of section 4.1 there is a class of spin-webs which have the property that a space deformation of a given spin-work results, generically, in an orthogonal spin-web space. 


\subsection{Averaging over the diffeomorphism group}

In [4] the spin-networks of the analytic category can be employed to construct solutions to the diffeomorphism constraint $\dagger$. The construction consists of averaging the state over the diffeomorphisms of $\Sigma$. With the Baez-Sawin results [17] the averaging was easily extended to averaging of a spin-network state with respect to the smooth diffeomorphisms [20,24]. Our goal now is to extend that construction to a spin-web state.

We need first some notation.

Given a labelled web $(w, j)$ the gauge group acts in $V_{w, j}$ at the end points $x, y$ of an edge $p$ through its action on the parallel transport along the edges,

$$
U_{e} \mapsto g(y)^{-1} U_{p} g(x)
$$

where the $G$-valued function $g: \Sigma \rightarrow G$ defines an element of the group of the gauge transformations. The end points of the edges of a web will be called vertices in the following.

One can further decompose each $V_{w, j}$ into the irreducible representation $V_{w, j, l, c}$, of that action, assigning to each vertex $v$ of the web $w$ an irreducible representation $l$ and an extra label $c$, because several equivalent but mutually orthogonal representations can appear more then once (see $[4,15,16]$ for the details about the gauge-invariant spin-networks and [21-23]). The quadruple $(w, j, l, c)$ will also be called a labelled web.

As in [4] an important notion will be the group of trivial action diffeomorphisms of a web whose vertices are labelled by irreducible representations. We define it as follows. Given a web $w_{0}$ fix a labelling $l_{0}$ of its vertices by the irreducible representations. The group $\operatorname{TA}\left(w_{0}, l_{0}\right)$ is the subgroup of those diffeomorphisms of $\Sigma$ which act trivially in the space $V_{w_{0}, j, c, l_{0}}$ for every labelled web $\left(w_{0}, j, l_{0}, c\right)$ with the fixed web $w_{0}$ and the labelling $l_{0}$, and with arbitrary labellings $j, c$. In fact, the group depends quite weakly on $l_{0}$. If the representation $l_{0}(v)$ is non-trivial for every vertex $v$, then the group $\operatorname{TA}\left(w_{0}, l_{0}\right)$ consists of those diffeomorphisms which preserve every edge (including its orientation) of $w_{0}$. However, there are some more diffeomorphisms acting 'trivially' from the above point of view. Consider a 2-valent vertex $v_{0}$ of the web $w_{0}$ which is the intersection point of two edges $e_{1}, e_{2} \in w$ such that, modulo a change of orientation, one edge is a smooth extension of the other. Suppose $l_{0}\left(v_{0}\right)$ is the trivial representation. Then, every element of the associated space $V_{w_{0}, j, c, l_{0}}$, as a function on $\mathcal{A}$, depends on the connection $A$ through the parallel transport $U_{e_{2} \circ e_{1}-1}$. Therefore, in this case, every diffeomorphism preserving the oriented non-parametrized curve $e_{2} \circ e_{1}^{-1}$ is an element of $\operatorname{TA}\left(w_{0}, l_{0}\right)$.

The group of smooth diffeomorphisms of $\Sigma$ acts naturally in the space of the cylindrical functions Cyl. Given a diffeomorphism $\varphi$ and $\Psi \in$ Cyl denote the result of the action by $\varphi \Psi$. Consider a labelled web $(w, j, l, c)$, and $\Psi \in V_{w, j, l, c}$. We attempt to define an averaged state $\left\langle\left.\Psi\right|_{\text {av }}\right.$ by the following action on a cylindrical function $f \in \mathrm{Cyl}$ :

$$
\langle\Psi|(f):=\frac{1}{\kappa(w, j, l)} \sum_{[\varphi] \in \operatorname{Diff}(\Sigma) / \mathrm{TA}(w, l)}(\varphi \Psi \mid f),
$$

where $\kappa(w, j, l)$ is a constant. The following two conditions should be satisfied by the averaging:

(a) finiteness, given $\Psi$ the result should be finite for a large class of $f \in \mathrm{Cyl}$;

(b) consistency, since the spin-web spaces are not orthogonal to each other, we have to ensure that given two different labelled webs $(w, j)$ and $\left(w^{\prime}, j^{\prime}\right)$ and a state $\Psi \in V_{w, j} \cap V_{w^{\prime}, j^{\prime}}$ our definition of the averaging gives the same result regardless of which of the above webs we use.

$\dagger$ That is, the diffeomorphism-invariant state which are elements of the space $\mathrm{Cyl}^{\prime}$, the topological dual of Cyl. 
Suppose $f$ is contained in the subspace $V_{w^{\prime}, j^{\prime}}$ associated with some labelled web $\left(w^{\prime}, j^{\prime}\right)$ and that $j^{\prime}\left(e^{\prime}\right) \neq 0$ for every $e^{\prime} \in w^{\prime}$. Now, owing to theorem 4.1 , the only non-zero terms in (4.18) come from the diffeomorphisms such that both $\varphi(R(w))=R(w)$ and the webs $w$ and $w^{\prime}=\varphi(w)$ satisfy property (c) of theorem 4.1. Define the symmetry group $\operatorname{SG}(w, l)$ of a labelled web $(w, j, l, c)$ to be

$\operatorname{SG}(w, l)=\left\{[\varphi] \in \operatorname{Diff}(\Sigma) / \operatorname{TA}(w, l) \mid \varphi(R(w))=R(w) \quad\right.$ and $\quad w^{\prime}:=\varphi(w)$ satisfies

(c) of theorem 4.1$\}$.

What we need to assume is that $\operatorname{SG}(w, l)$ is finite. A general cylindrical function can always be written as an infinite sum,

$$
f=\sum_{j^{\prime}} a_{j^{\prime}} f_{j^{\prime}}, \quad f_{j^{\prime}} \in V_{w^{\prime}, j^{\prime}}
$$

with respect to the labellings $j^{\prime}$ of the edges of a single web $w^{\prime}$. To see that also for a general cylindrical function $f$ the sum (4.18) is finite as long as the symmetry group $\operatorname{SG}(w, l)$ is finite, consider $[\varphi]$ in the sum (4.18) such that

$$
(\varphi \Psi \mid f) \neq 0 \text {. }
$$

The projection of $\varphi \Psi$ onto $V_{w^{\prime}, j^{\prime}}$ is not trivial only for a finite number of the labellings $j^{\prime}$. (To see that, take $w^{\prime} \geqslant w$, to represent $f$.)

Turn now to the consistency condition (b). It will constrain the freedom of the constants $\kappa(w, j, l)$. Consider a labelled web $\left(w_{0}, j_{0}, l_{0}, c_{0}\right)$ and a state

$$
\left|w_{0}, j_{0}, l_{0}, c_{0}, M_{0}\right\rangle \in V_{w_{0}, j_{0}, l_{0}, c_{0}}
$$

which satisfies an identity

$a_{0}\left|w_{0}, j_{0}, l_{0}, c_{0}, M_{0}\right\rangle=a_{1}\left|w_{1}, j_{1}, l_{1}, c_{1}, M_{1}\right\rangle+\cdots+a_{r}\left|w_{r}, j_{r}, l_{r}, c_{r}, M_{r}\right\rangle$.

Without loss of generality, we assume that the identity cannot be obtained as a sum of two non-trivial identities. That is we may assume that $|w, j, l, c, M\rangle$ on the left-hand side is not orthogonal to any of the terms on the right-hand side. Therefore, the web $w$ is in relation (c) of theorem 4.1 with each of the webs $w_{1}, \ldots, w_{r}$ on the right-hand side. Moreover, if $l_{s}\left(v_{s}\right) \neq 0$ for any of the vertices $v_{s}$ of any of the webs above, then the point $v_{s}$ is a vertex of every one of the webs in the identity, and

$$
l_{1}\left(v_{s}\right)=\cdots=l_{r}\left(v_{s}\right) .
$$

We may also write the identity in such a way that the labelled webs $\left(w_{0}, j_{0}\right), \ldots,\left(w_{r}, j_{r}\right)$ are all different.

To ensure that the averaging is consistent with the identity we observe the following.

Lemma 4.4. Suppose a labelled spin-web $(w, j, l, c)$ is non-degenerate. If $\left(w^{\prime}, j^{\prime}, l^{\prime}, c^{\prime}\right)$ is another labelled spin-web such that the corresponding spin-web spaces $V_{w, j, l, c}$ and $V_{w^{\prime}, j^{\prime}, l^{\prime}, c^{\prime}}$ are not orthogonal to each other, then the corresponding groups of the trivially acting diffeomorphisms coincide,

$$
\operatorname{TA}(w, l)=\operatorname{TA}\left(w^{\prime}, l^{\prime}\right)
$$

Proof of lemma 4.4. We again take advantage of theorem 4.1. Let $w^{\prime \prime}$ be the web given by theorem 4.1 which satisfies (c) of that theorem. Consider the following labelling $l^{\prime \prime}$ of the vertices of $w^{\prime \prime}$ with the irreducible representations. For a vertex $v$ of $w^{\prime \prime}$ set

$$
l^{\prime \prime}(v)= \begin{cases}l(v), & \text { if } v \text { is a vertex of } w, \\ 0, & \text { otherwise. }\end{cases}
$$


Recall that every vertex $v$ of $w^{\prime \prime}$ which is not a vertex of $w$ is obtained by subdividing an edge of $w$. It is easy to check that the corresponding groups of trivially acting diffeomorphisms coincide,

$$
\mathrm{TA}(w, l)=\operatorname{TA}\left(w^{\prime \prime}, l^{\prime \prime}\right) .
$$

However, it is also true for $\operatorname{TA}\left(w^{\prime}, l^{\prime}\right)$ and $\operatorname{TA}\left(w^{\prime \prime}, l^{\prime \prime}\right)$, which completes the proof of lemma 4.4.

Equation (4.18) for the averaging depends on the group $\operatorname{TA}(w, l)$ of a given labelled web $(w, j, l, c)$ and on the unspecified constant $\kappa(w, j, l)$. For every term in the identity (4.23) the group of the trivially acting diffeomorphisms is the same, and equals $\operatorname{TA}\left(w_{0}, l_{0}\right)$. Therefore, to ensure consistency, we have to take the constants $\kappa(w, j, l)$ depending only on $\operatorname{TA}(w, l)$,

$$
\kappa(w, j, l)=f(\operatorname{TA}(w, l)) .
$$

A quite natural choice is for example

$$
\kappa(w, j, l)=|\mathrm{SG}(w, l)|,
$$

where $|\mathrm{SG}(w, l)|$ is the number of elements of the symmetry group of a labelled web.

Let us summarize our results: denote by $\mathrm{Cyl}_{\text {nondeg }}$ and $\mathrm{Cyl}_{\mathrm{deg}}$ the subspaces of Cyl spanned by the spin-web subspaces corresponding to, respectively, non-degenerate and degenerate labelled spin-webs. Next, let $\mathrm{Cyl}_{\text {nondegfin }}$ and $\mathrm{Cyl}_{\text {nondeginf }}$ be spanned by the spin-web spaces corresponding to non-degenerate labelled webs of, respectively, finite and infinite symmetry groups.

Theorem 4.2. The Hilbert space $\mathcal{H}$ of all the square-integrable functions can be orthogonally decomposed in the following way:

$$
\mathcal{H}=\overline{\mathrm{Cyl}_{\text {nondeg }}} \oplus \overline{\mathrm{Cyl}_{\mathrm{deg}}}=\overline{\mathrm{Cyl}_{\text {nondegfin }}} \oplus \overline{\mathrm{Cyl}_{\text {nondeginf }}} \oplus \overline{\mathrm{Cyl}_{\mathrm{deg}}},
$$

where the overline denotes the Hilbert closure; the diffeomorphism averaging given by (4.18) and (4.28) defines a linear map

$$
\mathrm{Cyl}_{\text {nondegfin }} \ni \Psi \quad \mapsto\langle\Psi| \in \mathrm{Cyl}^{\prime},
$$

with $\mathrm{Cyl}^{\prime}$ the topological dual to the space $\mathrm{Cyl}$.

The proof follows from the above arguments, theorem 4.1 and lemma 4.3.

Some remarks are in order about the subspaces $\mathrm{Cyl}_{\text {nondeginf }}$ and $\mathrm{Cyl}_{\mathrm{deg}}$ which are excluded from the domain of the averaging map.

The first subspace is given by non-degenerate spin-webs with infinite symmetry groups. An example of such a spin-web was given in [20]. The spin-web considered there is degenerate in terms of the present paper, but it is not hard to modify it to a non-degenerate spin-web of the infinite symmetry group. The idea behind the construction of such an example is that a part of a web can be non-trivially mapped into itself by a diffeomorphism which preserves the rest of the web. If similar parts emerge infinitely many times (in every neighbourhood of a base of a tassel) then we have infinitely many diffeomorphisms, which are symmetries of the web.

The second group of states is given by degenerate labelled webs $(w, j)$. An example of such a web is shown in figure 1. In all the examples of degenerate labelled webs we are aware of, an associated cylindrical function can be decomposed into a converging infinite sum of cylindrical functions, each being associated with a web obtained from the original one by removing a (degenerate) segment (see section 5.3).

From the point of view of a diffeomorphism-invariant theory of connections, the averaged states are solutions of the diffeomorphism constraint. The action of the Hamiltonian constraint operator of the gravitational field can be extended to those states. We return to that issue in section 6 . 


\section{Diffeomorphism-invariant operators}

In the analytic category [4], the Hilbert space of cylindrical functions is orthogonally decomposed into subspaces labelled by graphs in such a way that every diffeomorphisminvariant operator preserves the subspaces. The aim of this subsection is to show that in the smooth case, certain subspaces are also necessarily preserved by diffeomorphism-invariant operators. However, the result that we derive below is not as strong as in the analytic category.

\subsection{Ranges of diffeomorphism-invariant operators}

Given a web $w$ denote by $\mathcal{H}_{R(w)}$ the Hilbert spaces obtained by using all the cylindrical functions which are associated with the webs whose ranges are contained in the range of $w$,

$$
\mathcal{H}_{R(w)}:=\overline{\sum_{w^{\prime} \mid R\left(w^{\prime}\right) \subset R(w)} \mathcal{H}_{w^{\prime}}}
$$

Theorem 5.1. Suppose $\mathcal{O}$ is a diffeomorphism-invariant operator defined in a domain $\mathcal{D}(\mathcal{O})$ in the Hilbert space $\mathcal{H}=L^{2}\left(\mathcal{A}, \mathrm{d} \mu_{0}\right)$; then,

(a) for every web $w$, the Hilbert subspace $\mathcal{H}_{R(w)}$ associated with its range is preserved by the operator $\mathcal{O}$;

(b) in particular, when a web is a graph, then the space

$$
\oplus_{j} V_{w, j}
$$

$j$ running over all the labellings of the edges of $w$ by the irreducible (possibly trivial) representations, is preserved by the operator $\mathcal{O}$;

(c) suppose that, for every labelled web $\left(w^{\prime}, j^{\prime}\right)$, it is true that, $V_{w^{\prime}, j^{\prime}} \subset \mathcal{D}\left(\mathcal{O}^{\dagger}\right)$; let $\Psi \in V_{w, j} \cap \mathcal{D}(\mathcal{O})$ and $\mathcal{N} \in V_{w^{\prime}, j^{\prime}}$ where $(w, j)$ are labelled webs; then,

$$
(\mathcal{O} \Psi \mid \mathcal{N}) \neq 0 \quad \Rightarrow \quad R\left(w^{\prime}\right)=R(w),
$$

provided the labellings $j, j^{\prime}$ do not assign the trivial representation to any of the edges of the webs $w$ and, respectively, $w^{\prime}$; in particular, if the web $w$ is a graph, then the operator preserves the space

$$
\oplus_{j} V_{w, j},
$$

$j$ running through all the labellings of the edges of $w$ by irreducible and non-trivial representations.

Proof. Consider a web $w$ and a state $\Psi \in \mathcal{H}_{R(w)} \cap \mathcal{D}(\mathcal{O})$. For the proof of (a), it is enough to show that for every labelled web $\left(w^{\prime}, j^{\prime}\right)$ such that $w^{\prime} \geqslant w$ containing an edge $e^{\prime} \in w^{\prime}$ such that the range $R\left(e^{\prime}\right)$ is not contained in $R(w)$

$$
j^{\prime}\left(e^{\prime}\right) \neq 0 \Rightarrow \mathcal{O} \Psi \perp V_{w^{\prime}, j^{\prime}}
$$

(Notice, that the subspaces $V_{w^{\prime}, j^{\prime}}$ span a dense subspace in the whole of $\mathcal{H}$, even when we restrict ourselves, to $w^{\prime} \geqslant$ a given $w$ because we allow $j^{\prime}$ to contain trivial representations.) Let $e_{0}^{\prime} \in w^{\prime}$ be an edge such that the range $R\left(e_{0}^{\prime}\right)$ is not contained in $R(w)$. In $e_{0}^{\prime}$, there is a regular point $x_{0}^{\prime}$ of the web $w^{\prime}$ which is not contained in $R(w)$. According to the definition of a tassel, the edge $e_{0}^{\prime}$ contains a sequence of the regular points $\left(x_{k}^{\prime}\right)_{k=0}^{\infty}$ such that each of the points has the same type as $x_{0}^{\prime}$, and the sequence converges to the beginning point of $e_{0}^{\prime}$, the base point of the corresponding tassel. For each of the points $x_{k}^{\prime}$ take an open neighbourhood $\mathcal{U}_{k} \ni x_{k}^{\prime}$ diffeomorphic to a ball, such that the intersection $R\left(w^{\prime}\right) \cap \mathcal{U}_{k}$ is an embedded open interval, say $s_{k}^{\prime}$. Consider a diffeomorphism $\varphi \in$ Diff such that: 
(a) $\varphi$ preserves each of the $\mathcal{U}_{k}$ and acts trivially outside of $\cup_{k} \mathcal{U}_{k}$;

(b) $\varphi\left(x_{k}^{\prime}\right) \neq x_{k}^{\prime}$, for every point of the sequence;

(c) $\varphi$ deforms each segment $s_{k}^{\prime}$ in the same way, modulo diffeomorphisms; that is, for every pair of the segments $s_{k}^{\prime}$ and $s_{l}^{\prime}$, the pair $\left(s_{k}^{\prime}, \varphi\left(s_{k}^{\prime}\right)\right)$ is diffeomorphic to $\left(s_{l}^{\prime}, \varphi\left(s_{l}^{\prime}\right)\right)$.

Let $e_{1}^{\prime}, \ldots, e_{m}^{\prime}$ be the edges of $w^{\prime}$ which overlap the edge $e_{0}^{\prime}$ at $x_{0}$. Hence, they overlap it at each of the points $x_{1}, \ldots, x_{k}, \ldots$ It follows from (a)-(c) above, that the family of edges obtained from $w^{\prime}$ and its deformation, namely

$$
w^{\prime \prime}:=w^{\prime} \cup \varphi\left(w^{\prime}\right)=\left\{\varphi\left(e_{0}^{\prime}\right), \varphi\left(e_{1}^{\prime}\right), \ldots, \varphi\left(e_{m}^{\prime}\right)\right\} \cup w^{\prime},
$$

is also a web.

Now, given a labelling $j^{\prime}$ of $w^{\prime}$, we can trivially extend it to a labelling $j^{\prime \prime}$ of $w^{\prime \prime}$ by setting

$$
j^{\prime \prime}\left(\varphi\left(e_{0}^{\prime}\right)\right)=\cdots=j^{\prime \prime}\left(\varphi\left(e_{m}^{\prime}\right)\right)=0 .
$$

On the other hand, the diffeomorphism $\varphi$ carries $j^{\prime}$ into another labelling $\varphi\left(j^{\prime}\right)$ of the web $w^{\prime \prime}$ given by $\varphi\left(j^{\prime}\right) \varphi\left(e^{\prime}\right):=j^{\prime}\left(e^{\prime}\right)$ for every edge of $w^{\prime}$. Obviously we then have

$$
j^{\prime}\left(e_{0}^{\prime}\right) \neq 0 \Rightarrow \varphi\left(j^{\prime}\right) \neq j^{\prime \prime} .
$$

Therefore, if $j^{\prime}$ satisfies (5.8) then for every $\mathcal{N} \in V_{w^{\prime}, j^{\prime}}$, the functions $\mathcal{N}$ and $\varphi(\mathcal{N})$ are orthogonal to each other. However, the diffeomorphism acts trivially on $\Psi$. Therefore, from the diffeomorphism invariance of $\mathcal{O}$, the projection of $\mathcal{O} \Psi$ on $\mathcal{N}$ is $\varphi$ invariant,

$$
(\mathcal{O} \Psi \mid \varphi \mathcal{N})=\left(\varphi^{-1} \mathcal{O} \Psi \mid \mathcal{N}\right)=\left(\mathcal{O} \varphi^{-1} \Psi \mid \mathcal{N}\right)=(\mathcal{O} \Psi \mid \mathcal{N})
$$

Moreover, it is easy to iterate the above construction to obtain an infinite family of diffeomorphisms each of which satisfies the conditions (a)-(c) above and such that for every pair of this set of diffeomorphisms,

$$
\varphi^{\prime} \neq \varphi \quad \text { it follows that }\left(\varphi^{\prime} \mathcal{N}_{w^{\prime \prime}, p^{\prime \prime}} \mid \varphi \mathcal{N}_{w^{\prime \prime}, p^{\prime \prime}}\right)=0 .
$$

If $\varphi$ varies through all possible diffeomorphisms like this, the set of the resulting states $\varphi \mathcal{N}$ has an infinite number of mutually orthogonal elements. Since a densely defined operator cannot have an infinite number of non-vanishing matrix elements of the same value it follows that

$$
\left(\mathcal{O} \Psi \mid \varphi \mathcal{N}_{w^{\prime \prime}, j^{\prime \prime}}\right)=0 \text {. }
$$

This is sufficient to complete the proof of part (a) of the statement.

If $w$ is a graph, then $R\left(w^{\prime}\right) \subset R(w)$ implies that $w^{\prime}$ is also a graph. Let $v^{\prime}$ be a vertex of $w^{\prime}$ which is not a vertex of the graph $w$. Therefore, it is contained in the interior of an edge $e \in w$. For every labelling $\left(w^{\prime}, j^{\prime}, l^{\prime}, c^{\prime}\right)$ of the graph $w^{\prime}$ such that $l^{\prime}\left(v^{\prime}\right) \neq 0$, and for every spin-web state $\mathcal{N} \in V_{w^{\prime}, j^{\prime}, l^{\prime}, c^{\prime}}$, we prove that

$$
(\mathcal{O} \Psi \mid \mathcal{N})=0
$$

in the same way as before. We construct an infinite class of the states diffeomorphic to $\mathcal{N}$ and orthogonal to each other. The diffeomorphisms we use move the vertex $v^{\prime}$ along the edge $e$ but preserve the edges of the web $w$, hence they also preserve the function $\Psi$. This completes the proof of part (b). Point (c) follows easily from (a) and (b) and their applications to the adjoint operator $\mathcal{O}^{\dagger}$. This completes the proof of theorem 5.1. 


\subsection{Strongly degenerate labelled webs}

In the previous section we have split the Hilbert space into the orthogonal sum of degenerate and non-degenerate spin-web states and the last sector has again been decomposed orthogonally with respect to possible symmetry groups of the labelled spin-webs. The diffeomorphism averaging is well defined only in one of those sectors, namely in the subspace $\mathrm{Cyl}_{\text {nondegfin }}$ spanned by all the spin-web states of non-degenerate labelled webs which have at most finite symmetry groups. For this reason it is natural to ask whether a diffeomorphism-invariant operator is necessarily consistent with that decomposition and whether it preserves the domain of the diffeomorphism averaging.

It is easy to see that, owing to theorem 5.1 (c), a symmetric diffeomorphism-invariant operator preserves the finiteness of the symmetry groups. Regarding the non-degeneracy, we will show below that the image of a non-degenerate spin-web state under the action of a diffeomorphism-invariant operator is orthogonal to what we will define as 'strongly degenerate' states. We will also indicate a large class of webs for which the degeneracy is equivalent to the 'strong degeneracy', and we will conjecture that the equivalence holds for all the spin-webs.

Given an edge $e$ let

$$
\hat{p}_{e}: \mathcal{H} \rightarrow \mathcal{H}
$$

be the projection onto the subspace given by the cylindrical functions independent of the holonomy along $e$. Explicitly, the projection can be defined as follows. Given a cylindrical function $\Psi \in \mathrm{Cyl}$ take a web $w \geqslant\{e\}$ such that $\Psi \in \mathcal{H}_{w}$. Let $e_{1}, \ldots, e_{k} \in w$ be all those elements of $w$ which are segments of $e$. We will use the orthogonal projection,

$$
\hat{p}_{e, w}: \quad \mathcal{H}_{w} \rightarrow \oplus_{j: j\left(e_{1}\right)=\cdots=j\left(e_{k}\right)=0} V_{w, j},
$$

to define

$$
\hat{p}_{e} \Psi=\hat{p}_{e, w} \Psi
$$

A labelled web $(w, j)$ is strongly degenerate if there is an infinite sequence of disjoint regular segments $s_{1}, \ldots, s_{l}, \ldots$, such that for every $\Psi \in V_{w, j}$

$$
\lim _{l \rightarrow \infty}\left(1-\hat{p}_{s_{1}}\right) \cdots\left(1-\hat{p}_{s_{l}}\right) \Psi=0,
$$

in the Hilbert space topology.

Indeed, the strong degeneracy implies the weak degeneracy. A labelled web $(w, j)$ is either degenerate or non-degenerate. If it is non-degenerate, then for every regular segment $s$

$$
\hat{p}_{s}\left(V_{w, j}\right)=0,
$$

and the left-hand side of (5.16) is $\Psi$ itself for every $\Psi \in V_{w, j}$.

Theorem 5.2. Suppose $\mathcal{O}$ is a diffeomorphism-invariant operator in $\mathcal{H}$ such that the domain $\mathcal{D}\left(\mathcal{O}^{\dagger}\right)$ contains all the spin-web subspaces $V_{w^{\prime}, j^{\prime}}$. Then, for every non-degenerate spin-web state $\Psi$ and every strongly degenerate labelled spin-web state $\mathcal{N}$,

$$
(\mathcal{O} \Psi \mid \mathcal{N})=0 .
$$

Proof. It follows from the non-degeneracy of $\Psi$ and from theorem 4.1 that any diffeomorphism $\varphi$ which deforms only the segment $s_{1}$ and acts trivially in $R(w) \backslash R\left(s_{1}\right)$ satisfies

$$
\left(\varphi \Psi \mid \mathcal{O}^{\dagger} \hat{p}_{s_{1}} \mathcal{N}\right)=\left(\Psi \mid \mathcal{O}^{\dagger} \hat{p}_{s_{1}} \mathcal{N}\right), \quad(\varphi \Psi \mid \Psi)=0 .
$$


So, there exists an infinite family of states $\varphi_{k} \Psi$, such that

$$
\begin{aligned}
& \left(\varphi_{k_{1}} \Psi \mid \mathcal{O}^{\dagger} \hat{p}_{s_{1}} \mathcal{N}\right)=\left(\varphi_{k_{2}} \Psi \mid \mathcal{O}^{\dagger} \hat{p}_{s_{1}} \mathcal{N}\right), \\
& \left(\varphi_{k_{1}} \Psi \mid \varphi_{k_{2}} \Psi\right)=0 .
\end{aligned}
$$

It follows that

$$
\left(\mathcal{O} \Psi \mid \hat{p}_{s_{1}} \mathcal{N}\right)=0 .
$$

This shows that

$$
(\mathcal{O} \Psi \mid \mathcal{N})=\left(\mathcal{O} \Psi \mid\left(1-\hat{p}_{s_{1}}\right) \mathcal{N}\right) .
$$

Next, using the same argument for the sequences $s_{2}, \ldots, s_{k}, \ldots$, we prove that for arbitrary $k$

$$
(\mathcal{O} \Psi \mid \mathcal{N})=\left(\mathcal{O} \Psi \mid\left(1-\hat{p}_{s_{k}}\right) \cdots\left(1-\hat{p}_{s_{1}}\right) \mathcal{N}\right) .
$$

Taking the limit $k \rightarrow 0$ and using the strong degeneracy of $\mathcal{N}$ completes the proof of theorem 5.2.

Our conjecture is:

Conjecture. Every degenerate state is strongly degenerate.

So if the conjecture is true, then every symmetric, diffeomorphism-invariant operator preserves the subspace $\mathcal{H}_{\text {nondegfin }}$ in which the diffeomorphism averaging has been well defined.

We will advocate the conjecture in the next subsection.

\subsection{A large class of strongly degenerate spin-webs}

There is a large class of webs, for which the degeneracy of a labelling does imply strong degeneracy. In this subsection we consider webs for which the following is true. For every tassel $T=\left\{e_{1}, \ldots, e_{m}\right\}$ of a given web $w$ and for a parametrization of the edges of $T$ given by point (b) of the definition of a tassel (say, the parametrization is defined on $[0,1]$ ) for every $0<t<1$ there is $0<t^{\prime}<t<1$ such that the segments $e_{1 t t^{\prime}}, \ldots, e_{m t t^{\prime}}$ of all the edges $e_{1}, \ldots, e_{k}$ are holonomically independent. (The above property is satisfied for the web in the example given in section 4.1. In fact, we do not know of any counter example, i.e. any web that would fail to satisfy this property.)

Now, consider a degenerate labelling $(w, j)$ of a web $w$ and a state $\Psi \in V_{w, j}$. We will calculate explicitly the action of the projection operator $\hat{p}_{s}$ for appropriately selected segments. Let $s$ be a regular segment, such that the tensor product $\rho_{j\left(e_{1}\right)} \otimes \cdots \otimes \rho_{j\left(e_{k}\right)}$ contains the trivial representation in the decomposition into the irreducible representations, where $e_{1}, \ldots, e_{k}$ are the edges of $w$ that overlap $s$. The degeneracy guarantees the existence of such a segment. Then, owing to the properties of a tassel, there is a segment $s_{0}$ of the same type as $s$ which is close enough to the base point of the tassel for the following to be true: if we write each of the edges overlapping at $s$ as

$$
e_{I}=e_{I+} \circ s \circ e_{I-}, \quad I=1, \ldots, k,
$$

then the segments $e_{1+}, \ldots, e_{k+}$ are holonomically independent (the beginning segments $e_{I-}$ are holonomically independent for any choice of $s$ by the very construction of a tassel). We will show, that

$$
\left\|\left(1-\hat{p}_{s}\right) \Psi\right\|=c\left(j\left(e_{1}\right), \ldots, j\left(e_{k}\right)\right)\|\Psi\| .
$$

The main point of this result is that the coefficient $c$ depends only on the labels assigned by the labelling $j$ to the edges. Of course the coefficient is less then one, because the operator is 
a projection and the trivial representation does appear in the decomposition above. The new function $\left(1-\hat{p}_{s}\right) \Psi$ can be written as

$$
\left(1-\hat{p}_{s}\right) \Psi=\sum_{n} \Psi_{n}
$$

where each $\Psi_{n}$ is again a spin-web state associated to a labelled web $\left(w_{n}, j_{n}\right)$ which contains a segment $s_{1}$ of the same properties as the segment $s_{0}$, namely

(a) $s_{1}$ is overlapped by $k$ edges, $e_{1}^{\prime}, \ldots, e_{k}^{\prime}$;

(b) the segments $e_{I}^{\prime}$ overlapping $s_{1}$ are labelled in the same way as the edges $e_{I}$,

$$
j_{n}\left(e_{I}^{\prime}\right)=j\left(e_{I}\right), \quad I=1, \ldots, k
$$

(c) the new segments $e_{1+}^{\prime}, \ldots, e_{k+}^{\prime}$ are holonomically independent (where $e_{I}^{\prime}=e_{I+}^{\prime} \circ s_{1} \circ e_{I-}^{\prime}$ ).

Therefore, we can use the result again to conclude that

$$
\left\|\left(1-\hat{p}_{s_{1}}\right)\left(1-\hat{p}_{s_{0}}\right) \Psi\right\|=c\left(j\left(e_{1}\right), \ldots, j\left(e_{k}\right)\right)^{2}\|\Psi\| .
$$

Finally, repeating the construction we find an infinite sequence of segments $s_{0}, s_{1}, \ldots, s_{N}, \ldots$, such that

$$
\left\|\left(1-\hat{p}_{s_{N}}\right) \cdots\left(1-\hat{p}_{s_{0}}\right) \Psi\right\|=c\left(j\left(e_{1}\right), \ldots, j\left(e_{k}\right)\right)^{N+1}\|\Psi\| .
$$

The sequence converges to 0 as $N \rightarrow \infty$, hence $\Psi$ is indeed strongly degenerate.

Let us now show that equation (5.26) is true. The function $\Psi$ can be written as

$$
\Psi=L_{A_{1} \cdots A_{k}} \mathcal{D}^{\left(j\left(e_{1}\right)\right)_{B_{1}}^{A_{1}}}\left(U_{e_{1}}\right) \cdots \mathcal{D}^{\left(j\left(e_{k}\right)\right)}{ }_{B_{k}}^{A_{k}}\left(U_{e_{k}}\right) R^{B_{1} \cdots b_{k}},
$$

where $L_{A_{1} \cdots A_{k}}, R^{B_{1} \cdots B_{k}} \in \mathrm{Cyl}_{w}$ do not involve any of the holonomies $U_{e_{1}}, \ldots, U_{e_{k}}$. Then, the action of the projection operator $\hat{p}_{s}$ on $\Psi$ is

$$
\begin{aligned}
& \hat{p}_{s} \Psi=L_{A_{1} \cdots A_{k}} \mathcal{D}^{\left(j\left(e_{1}\right)\right)}{ }_{C_{1}}^{A_{1}}\left(U_{e_{1+}}\right) \cdots \mathcal{D}^{\left(j\left(e_{k}\right)\right)}{ }_{C_{k}}^{A_{k}}\left(U_{e_{k+}}\right) c^{C_{1} \cdots C_{k}} \\
& c_{D_{1} \cdots D_{k}} \mathcal{D}^{\left(j\left(e_{1}\right)\right) D_{1}}\left({ }_{B_{1}}\left(U_{e_{1-}}\right) \cdots \mathcal{D}^{\left(j\left(e_{k-}\right)\right)}{ }_{B_{k}}^{D_{k}}\left(U_{e_{k}}\right) R^{B_{1} \cdots B_{k}} .\right.
\end{aligned}
$$

The comparison of the norms gives the following result:

$\left\|\hat{p}_{s} \Psi\right\|^{2}=c^{C_{1} \cdots C_{k}} \overline{c_{1}^{C_{1}^{\prime} \cdots C_{k}^{\prime}}} \delta_{C_{1} C_{1}^{\prime}} \cdots \delta_{C_{k} C_{k}^{\prime}} c_{D_{1} \cdots D_{k}} \overline{\bar{C}_{1}^{\prime} \cdots D_{k}^{\prime}} \delta^{D_{1} D_{1}^{\prime}} \cdots \delta^{D_{k} D_{k}^{\prime}}\|\Psi\|^{2}$.

From the orthogonality of $\hat{p}_{s} \Psi$ and $\left(1-\hat{p}_{s}\right) \Psi$ we conclude (5.26).

It is easy to see that as mentioned the web of the example in section 4.1 belongs to the class of webs considered in this section. Indeed, each of the two edges contains a segment which is not overlapped by the other edge. Interestingly, even if we use the range of the web to introduce more edges such that each regular segment is overlapped by more than one edge, in all the examples we considered we found only webs belonging to the same class. It is likely that for a semi-simple group this class contains all the webs.

What we have learnt from this section is that as in the case of the analytic category considered in [4], the algebra of the observables strongly commuting with the diffeomorphisms is reducible to the sectors labelled by the ranges of the webs. In the case when a web $w$ is a graph, the corresponding sector is $\mathcal{H}_{w}$. 


\section{The gravitational field operators}

For the quantization of gravity there are two kinds of operators that play a key role. The first kind consists of the so-called geometrical operators [23, 25, 27, 29] corresponding, respectively, to lengths, areas and volumes of curves, areas and volumes. The second kind consists of constraint operators $[4,28]$, most importantly the Hamiltonian constraint. These have been defined in the analytical category so far. We will now extend them to the smooth category.

\subsection{The 3-geometry operators}

The regularizations of the operators representing geometric functionals can be easily modified to the case of smooth curves. A problem one encounters then is that when a functional depending on $E$ is given by an integral over a surface or a region in $\Sigma$ the resulting operator often is not well defined in any dense domain. This means that a given surface or region is just infinite in certain states of the system.

To begin with, consider a functional associated with an oriented 2-surface $S$ (see [23, 25] for details)

$$
E_{S}^{i}=\int_{S} \frac{1}{2} E^{i a} \epsilon_{a b c} \mathrm{~d} x^{a} \wedge \mathrm{d} x^{b},
$$

and the corresponding quantum operator

$$
\hat{E}_{S}^{i}=\int_{S} \frac{1}{2} \frac{\mathrm{i} \delta}{\delta A_{a}^{i}} \epsilon_{a b c} \mathrm{~d} x^{a} \wedge \mathrm{d} x^{b} .
$$

Let $\Phi \in \mathrm{Cyl}_{w}$ where $w$ is a web. 'Generically', every edge $p$ of $w$ has only a finite number of isolated intersections with $S$ and only those contribute to its action on $\Phi$. Generally, however, that number is infinite. Every point $x$ of $e$ which is not contained in $S$ defines a segment $s$ of $e$ whose interior does not intersect $S$, whereas its ends lie on $S$ or coincide with the ends of $e$. However, for each edge $e$ the number of such segments is at most countable. Split each such segment into two pieces, oriented in such a way that they are outgoing from the surface and denote the resulting segments by $s_{e, 1}, \ldots, s_{e, k}, \ldots$.

Repeating the derivation of the first reference of [23] we see that for a cylindrical function $\Phi$ compatible with $w$, the only contribution comes from those end points $x_{e, I}$ of the segments $s_{e, I}$ which are contained in $S$, according to the formula

$$
\hat{E}_{S}^{i} \Phi=\frac{1}{2} \sum_{s_{e, I}} \kappa_{S}\left(s_{e, I}\right) J_{x_{e, I}, s_{e, I}}^{i} \Phi
$$

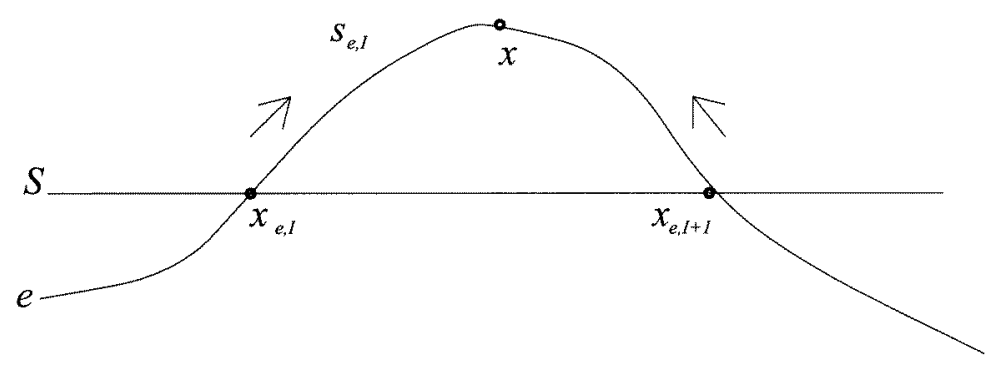

Figure 3. 
where $\kappa_{S}\left(s_{e, I}\right)$ is \pm 1 if the segment intersects $S$ and goes up/down (the above notation excludes segments which overlap $S$ and contribute zero). The operators $J_{x_{e, I}, s_{e, I}}^{i}$ were already defined in [23] where it is understood that we replace graphs by webs.

In the general case, for infinitely many intersections,

$$
\left\|\hat{E}_{S}^{i} \Phi\right\|=\infty
$$

Therefore, the domain of the operator is defined by the generic webs which intersect the surface $S$ finitely many times. Using the same notation, for the area of $S$ we obtain

$$
\widehat{\operatorname{area}} \Psi=\frac{1}{2} \ell_{P}^{2} \sum_{x_{e, I}} \sqrt{\left(\sum_{s} \kappa_{S}(s) J_{x_{e, I}, s}^{i}\right)\left(\sum_{s^{\prime}} \kappa_{S}\left(s^{\prime}\right) J_{x_{e, I}, s^{\prime}}^{i}\right)} \Psi,
$$

where $x_{e, I}$ runs through all the intersection points of the segments $s_{e, I}$ with $S$ and $s$ and $s^{\prime}$ run through all the segments $s_{e^{\prime}, I^{\prime}}$ which intersect the same $x_{e, I}$. However, again, the operator may take infinity. For instance, on a Wilson loop function

$$
\Psi(A)=\operatorname{Tr} U_{\alpha}(A)
$$

given by a non-self intersecting loop $\alpha$ which defines infinitely many, transversally intersecting the surface $S$ segments $s_{e, I}$ defined above, we have

$$
\widehat{\operatorname{area}} \Phi=\infty \Phi
$$

In the case of the volume of a region $R$ in $\Sigma$, the 'external' regularization of Rovelli and Smolin [25] does not appear to be easily applicable to the smooth curves case. On the other hand, the regularization of the second reference of [23] proposed for the volume operator can be extended to that case. Then, the contribution is given by all the points of intersection of three edges of $w$ such that the directions tangent to the edges are linearly independent and the intersection point is contained in $R$. The number of such generic intersection points is at most countable $\dagger$. The action of the resulting volume operator on a cylindrical function compatible with the web $w$ reads

$\hat{V}_{R} \Psi=\kappa_{o} \sum_{v} \sqrt{\left|\hat{q}_{v}\right|} \Psi \quad$ where $\quad \hat{q}_{v} \Psi=\frac{1}{48} \epsilon_{i j k} \sum_{s, s^{\prime}, s^{\prime \prime}} \epsilon\left(s, s^{\prime}, s^{\prime \prime}\right) J_{v, s}^{i} J_{v, s^{\prime}}^{j} J_{v, s^{\prime \prime}}^{k} \Psi$

where $v$ runs through all the intersection points between the edges of $w$ and each of $s, s^{\prime}, s^{\prime \prime}$ runs through all the segments intersecting at and are outgoing from $v$; the number $\epsilon\left(s, s^{\prime}, s^{\prime \prime}\right)$ equals $\pm 1,0$ depending on the orientation of the vectors tangent to $s, s^{\prime}, s^{\prime \prime}$ at $v$.

Similar statements apply to the length operator $\hat{L}(c)$ [27] for any piecewise-smooth curve $c$, since it uses the volume operator as a basic building block.

In summary, for all three operators considered above, the infinity arises as a sum of a countable number of finite terms. We can interpret this infinity as follows: let us first exclude most of the points giving rise to the infinity from the curve $c$, the surface $S$ or the region $R$, respectively. Then we will obtain a finite length, area or volume. Then, the process of gluing back the removed points one after the other can be interpreted as extending the curve, surface or region to (spatial) infinity.

$\dagger$ Indeed, for any given triple of edges $p, p^{\prime}, p^{\prime \prime}$ the generic intersection points are isolated from each other, so their set is countable; the number of triples is finite, determined by the number of edges of the web. 


\subsection{Hamiltonian constraint}

In section 4.2 we have defined new solutions of the diffeomorphism constraint. They were, however, so far not shown to be in the domain of the dual of the loop Hamiltonian constraint, as was defined in [28]. We observe below, that in fact the action of the dual $\hat{H}^{\prime}(N)$ of the Hamiltonian constraint can be naturally defined on them and that it is free of the infinities emerging in the case of the above 3-geometry operators.

One can easily repeat the regularization of [28] of the Hamiltonian constraint, with appropriate modifications, to the case of a web with an infinite number of intersection points among its defining curves. Recall, that in the Hilbert space $\mathcal{H}$ of the cylindrical functions, one first defines a regulator-dependent Hamiltonian operator $H_{\Delta}$, where $\Delta$ denotes the regulator. Its domain is no longer dense in the smooth category, in contrast to what happens in the analytical category. The reason for this is the same as in the case of the volume operator. Explicitly, the regulated Hamiltonian operator acts on a spin-web state $|\Gamma\rangle$ in the following way:

$$
\widehat{H(N)}_{\Delta}|\Gamma\rangle=\sum_{v} N(v) \hat{h}_{\Delta(v)}|\Gamma\rangle
$$

where $N$ is a lapse function, $v$ runs through all the intersection points of the edges in a web and for each $v$ the operator $\hat{h}_{\Delta(v)}$ is well defined for all the spin-web states. The number of intersection points is in general infinite, hence the total operator (6.9) is not well defined on such states. (The operator $\hat{h}_{\Delta(v)}$ involves the volume operator in such a way that, as in the volume operator case, only the triples of generically intersecting segments contribute.) Remarkably, however, the dual operator $\widehat{H(N)}_{\Delta}^{\prime}$ is well defined for any dual spin-web state, that is if $\Psi \in V_{w, j}$ and $\Psi_{1} \in V_{w_{1}, j_{1}}$ for labelled webs $(w, j)$ and $\left(w_{1}, j_{1}\right)$, then $\left(\Psi \mid \widehat{H(N)}{ }_{\Delta} \Psi_{1}\right)$ is finite.

Finally, the regulator-free dual Hamiltonian operator $\hat{H}^{\prime}(N)$ is well defined on the diffeomorphism-averaged dual-spin web states $\langle\Gamma|$, provided that we restrict ourselves to the space $\mathrm{Cyl}_{\text {nondegfin }}$ on which the diffeomorphism averaging has been defined in section 4.2. More

specifically, whenever $\Gamma \in \mathrm{Cyl}_{\text {nondegfin }}$ then for any lapse function the functional $\langle\Gamma| \hat{H}^{\prime}(N)$ is a well defined linear functional on the entire space $\mathrm{Cyl}_{\text {nondegfin }}$.

\section{Acknowledgments}

The authors thank Abhay Ashtekar, John Baez and Steve Sawin for helpful discussions and correspondence. JL was supported by Alexander von Humboldt-Stiftung and the Polish Committee on Scientific Research (KBN, grant no 2 P03B 017 12).

\section{References}

[1] Ashtekar A 1986 Phys. Rev. Lett. 572244 Ashtekar A 1987 Phys. Rev. D 361587

[2] Jacobson T and Smolin L 1988 Nucl. Phys. B 299295 Rovelli C and Smolin L 1990 Nucl. Phys. B 33180

[3] Gambini R, Griego J and Pullin J 1998 Nucl. Phys. B 534675

[4] Ashtekar A, Lewandowski J, Marolf D, Mourão J and Thiemann T 1995 J. Math. Phys. 36 519-51

[5] Ashtekar A and Isham C J 1992 Class. Quantum Grav. 91433

[6] Ashtekar A and Lewandowski J 1994 Representation theory of analytic holonomy $C^{\star}$ algebras Knots and Quantum Gravity ed J Baez (Oxford: Oxford University Press)

[7] Ashtekar A and Lewandowski J 1995 J. Geom. Phys. 17191

[8] Ashtekar A and Lewandowski J 1995 J. Math. Phys. 362170 
[9] Baez J 1994 Lett. Math. Phys. 31 213-23

Baez J 1994 Proc. Conf. on Quantum Topology ed D Yetter (Singapore: World Scientific)

(Baez J 1993 Diffeomorphism invariant generalized measures on the space of connections modulo gauge transformations Preprint hep-th/9305045)

[10] Marolf D and Mourão J M 1995 On the support of the Ashtekar-Lewandowski measure Commun. Math. Phys. 170 583-606

[11] Ashtekar A, Lewandowski J, Marolf D, Mourão J and Thiemann T 1994 A manifestly gauge invariant approach to quantum theories of gauge fields Geometry of Constrained Dynamical Systems ed J Charap (Cambridge: Cambridge University Press)

Ashtekar A, Lewandowski J, Marolf D, Mourão J and Thiemann T 1999 Osterwalder-Schrader-reconstruction and diffeomorphism invariance Preprint AE1-109 quant-ph/9904094

[12] Ashtekar A, Lewandowski J, Marolf D, Mourão J and Thiemann T 1997 J. Math. Phys. 38 5453-82

[13] Rovelli C and Smolin L 1990 Nucl. Phys. B 33180

[14] Rovelli C 1997 Loop quantum gravity J. Living Rev. 1998-1

[15] Rovelli C and Smolin L 1995 Phys. Rev. D 535743

[16] Baez J 1996 Spin network states in gauge theory Adv. Math. 117253

Baez J 1996 Spin networks in non-perturbative quantum gravity The Interface of Knots and Physics ed L Kauffman (Providence, RI: American Mathematical Society)

[17] Baez J and Sawin S 1997 J. Func. Anal. 1501

[18] Tits 1972 J. Algebra 20

[19] Thiemann T 1998 Class. Quantum Grav. 151207

[20] Baez J and Sawin S 1998 J. Func. Anal. 158253

[21] Thiemann T 1998 J. Math. Phys. 391236

[22] Thiemann T 1998 J. Math. Phys. 393347

[23] Ashtekar A and Lewandowski J 1997 Class. Quantum Grav. 14 A55 Ashtekar A and Lewandowski J 1997 Adv. Theor. Math. Phys. 1388

[24] Lewandowski J and Marolf D 1998 Int. J. Mod. Phys. D 7299

[25] Rovelli C and Smolin L 1995 Discreteness of volume and area in quantum gravity Nucl. Phys. B 442593 Rovelli C and Smolin L 1995 Nucl. Phys. B 456734 (erratum)

[26] De Pietri R and Rovelli C 1996 Phys. Rev. D 542664

[27] Thiemann T 1998 J. Math. Phys. 393372

[28] Thiemann T 1998 Class. Quantum Grav. 15 839-73

[29] Loll R 1995 Phys. Rev. Lett. 753048

[30] Gambini R and Pullin J 1996 Class. Quantum Grav. 13 L125

[31] Reed M and Simon B 1984 Methods in Modern Mathematical Physics vol I Functional Analysis (New York: Academic) 